\title{
Volumetric Tomographic 3D Bioprinting of Heterocellular Bone- like Tissues in Seconds
}

Jenny Gehlen, Wanwan Qiu, Ralph Müller, Xiao-Hua Qin*

Institute for Biomechanics, ETH Zurich, Leopold-Ruzicka-Weg 4, 8093 Zurich, Switzerland

*E-mail: qinx@ethz.ch (corresponding author)

\section{Abstract}

3D bioprinting has emerged as a powerful tool for custom fabrication of biomimetic hydrogel constructs that support the differentiation of stem cells into functional bone tissues. Existing stem cell-derived in vitro bone models, however, often lack terminally differentiated bone cells named osteocytes which are crucial for bone homeostasis. Here, we report ultrafast volumetric tomographic photofabrication of centimeter-scale heterocellular bone models that enabled successful 3D osteocytic differentiation of human mesenchymal stem cells (hMSCs) within hydrogels after 42 days co-culture with human umbilical vein endothelial cell (HUVECs). It is hypothesized that after 3D bioprinting the paracrine signaling between hMSCs and HUVECs will promote their differentiation into osteocytes while recreating the complex heterocellular bone microenvironment. To this, we formulated a series of bioinks with varying concentrations of gelatin methacryloyl (GelMA) and lithium Phenyl(2,4,6-trimethylbenzoyl)phosphinate (LAP). A bioink comprising 5\% GelMA and 0.05\% LAP was identified as an optimal material with high cell viability (>90\%) and excellent structural fidelity. Increasing LAP concentration led to much lower degree of cell spreading, presumably due to phototoxicity effects. Biochemical assays evidenced significantly increased expression of both osteoblastic markers (collagen-I, ALP, osteocalcin) and osteocytic markers (Podoplanin, PDPN; dentin matrix acidic phosphoprotein 1, Dmp1) after 3D co-cultures for 42 days. Additionally, we demonstrate volumetric 3D bioprinting of perfusable, pre-vascularized bone models where HUVECs selforganized into an endothelium-lined channel within 2 days. Altogether, this work leverages the benefits of volumetric tomographic bioprinting and 3D co-culture, offering a promising platform for scaled biofabrication of 3D bone-like tissues with unprecedented long-term functionality.

Keywords: volumetric tomographic bioprinting, bone tissue engineering, in vitro osteogenesis, endothelial co-culture, bioinks, pre-vascularization 


\section{Introduction}

The field of tissue engineering and regenerative medicine (TERM) aims to develop threedimensional (3D) cellular constructs that mimic physiological tissue structure and composition $[1,2]$. The idea of growing functional tissues in vitro holds the promise of revolutionizing future medicine by providing 'spare parts' for potential tissue replacements and drug screening for personalized treatments. 3D printing in combination with extracellular matrix (ECM)mimicking materials has fueled recent advances in tissue engineering of complex biomimetic niches and has emerged as a promising biomanufacturing technology [3, 4]. Even though 3D bioprinting techniques based on layer-by-layer deposition have extensively evolved during the last decade, printing duration, scaffold porosity and clinically relevant construct size remain major challenges $[5,6]$.

Volumetric 3D printing is a revolutionary technique based on tomographic light projections, for the fabrication of centimeter-scale constructs within tens of seconds [6-8]. This nozzle-free method leverages existing volumetric image modalities, such as computed tomography, to fabricate whole objects instantly and simultaneously. 3D light doses based on projections of multi-angular holographic light patterns cumulatively induce crosslinking of photoresponsive cell-laden bioinks with high spatial precision. The advantages of volumetric printing and its exceptionally high cell viability are especially relevant for applications in scalable biofabrication of living tissues. However, permissiveness and biocompatibility of employed hydrogel-based bioinks need to match the pace of cell differentiation and self-organization to eventually form a functional heterocellular tissue construct.

Stem cell-based tissue engineering has evolved rapidly and at present, osteogenic stimulants that drive the differentiation of mesenchymal stem cells (MSC) into osteoblasts are known [912]. In vivo differentiation is accompanied by matrix remodeling of the so-called osteoid, a hallmark of osteogenesis, starting with collagen deposition, alkaline phosphatase secretion and finally osteocalcin expression $[13,14]$. The expression of the osteoblastic markers Runt-related transcription factor 2 (RUNX2), collagen type I (COL1a2), alkaline phosphatase (ALPL), matrix metallopeptidase 14 (MMP14) and osteocalcin (BGLAP), as well as the osteocytic markers dentin matrix acidic phosphoprotein 1 (DMP1), podoplanin (PDPN) and sclerostin (SOST) is a hallmark in the dynamic osteoblast-to-osteocyte transition. The terminal osteocytic stage and the formation of networks with a complex three-dimensional lacuna-canalicular architecture remains a major challenge for state-of-the-art in vitro models [15]. Recent studies report the optimization of biophysical parameters and the use of different scaffold materials to 
promote bone formation, but so far, the most advanced 3D human bone model only resembles early osteogenesis with low level expression of osteocytic marker genes [9, 16-18].

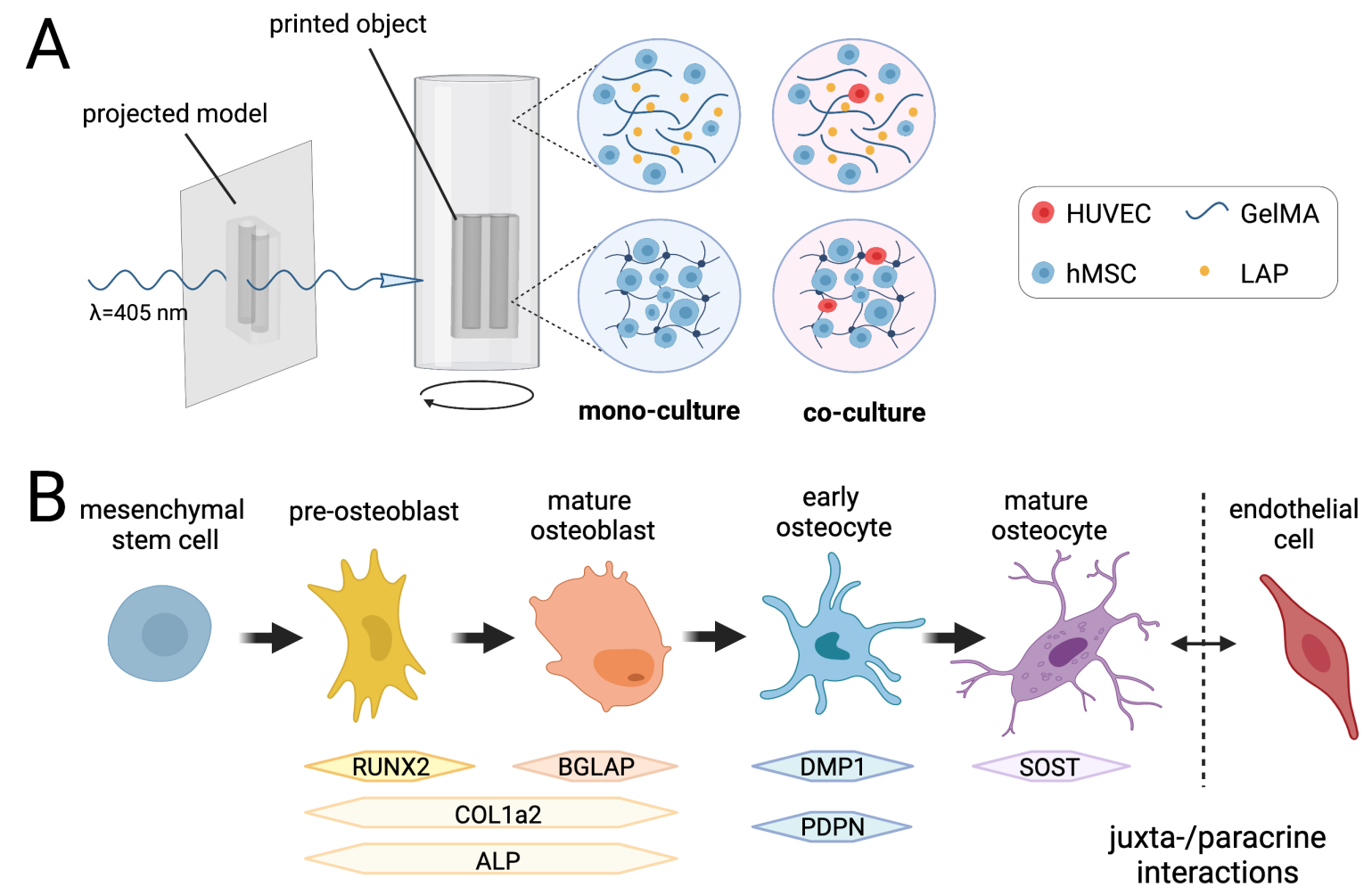

Figure 1: Illustration of volumetric tomographic bioprinting and enhanced stem cell osteogenic differentiation by endothelial co-culture. A) Schematic of volumetric bioprinting: Tomographic light projections lead to localized solidification of hydrogel constructs in a glass vial containing a cell suspension in a photo-crosslinkable bioink (GelMA/LAP). Mixed cell suspensions allow the fabrication of self-organized heterocellular constructs combining human mesenchymal stem cells (hMSC) with endothelial cells (HUVEC). B) Schematic of osteogenic differentiation from MSCs to mature osteocytes with respective marker gene expression. The juxta- and paracrine interactions between cells in the osteogenic lineage and endothelial cells promote osteogenic differentiation. Created with BioRender.com.

New concepts have to be explored to further enhance existing models of in vitro osteogenesis, one of them being a co-culture approach with endothelial cells. The lack of inter-cellular differentiation cues appeared as a major problem of in vitro cultures and heterocellularity can enhance optimized ECM scaffolds. Although the exact intercellular interactions remain poorly understood, a stimulating role of vascular endothelial growth factors (VEGF) in osteogenesis has been suggested [19]. VEGF is a signaling protein secreted by many cells that promotes the osteogenic differentiation of MSCs [20]. Additionally, other studies have shown the enhanced osteogenesis through endothelial co-cultures is associated with Wnt and bone morphogenic protein (BMP) signaling pathways [21]. Specifically, human umbilical vein endothelial cells (HUVEC) in combination with MSCs in the osteogenic lineage have been described as enhancers of osteoblast differentiation and function, based on their important role in the cellular 
communication network within the microenvironment of bone tissue [22, 23]. However, no long-term studies investigating the effect on terminal osteocytic differentiation have been conducted yet.

In this work, we report a new method to fabricate heterocellular bone-like tissues by leveraging the advantages of ultrafast volumetric tomographic bioprinting and 3D human MSC/HUVEC co-culture (Figure 1). Until now, only one publication reported volumetric bioprinting of cellladen hydrogel constructs using an bioink with 10\% GelMA [6]. The authors applied postcuring to improve the mechanical stability and structural complexity of printed constructs. Yet, it is unclear if these mechanically stiff environments are permissive for in vitro osteogenesis. Therefore, this study sought to provide an extensive functional assessment of 3D bioprinted heterocellular constructs by state-of-the-art cellular and molecular assays. First, a series of GelMA bioink compositions were extensively characterized with a focus on their printability, mechanical properties, and cell-compatibility. Furthermore, the effects of intercellular interactions on osteogenic differentiation were examined in a longitudinal analysis in both $2 \mathrm{D}$ and 3D over 42 days. Finally, the volumetric bioprinting technique was employed to create a prototype for a heterocellular pre-vascularized bone model where MSC-containing constructs with imprinted channel structures were lined with endothelial cells for future perfusion studies of pre-vascularized bone tissues.

\section{Materials and Methods}

\subsection{Biomaterials and volumetric 3D Printing}

\section{$\underline{\text { 2.1.1 Bioink preparation }}$}

All 3D constructs are based on gelatin methacryloyl (GelMA) which was synthesized following previously described protocols [24]. The degree of substitution (DS) of GelMA was estimated with ${ }^{1} \mathrm{H}-\mathrm{NMR}$ (Bruker Ultrashield, $400 \mathrm{MHz}$ ) in $\mathrm{D}_{2} \mathrm{O}$. Integration signal (2.87-3.00 ppm) in GelMA was compared to unmodified gelatin lysine integration signal (2.87-3.00 ppm). Phenylalanine signal (7.1-7.4 ppm) was used as internal reference. DS was found to be $\approx 57 \%$ using the following equation:

$\mathrm{DS}=\left[1-(\text { lysine methylene proton of GelMA)/(lysine methylene proton of gelatin) }]^{*} 100 \%\right.$

To obtain semi-sterile materials for aseptic cell culture techniques, synthesized GelMA was sterile filtered and lyophilized through polytetrafluoroethylene (PTFE) membranes of tissue culture flasks with a pore size of $0.22 \mu \mathrm{m}$. For photoinitiated crosslinking of functionalized methacryloyl groups, GelMA was dissolved in PBS containing the photoinitiator Lithium- 
Phenyl-2,4,6-trimethylbenzoylphosphinat (LAP) in different concentrations ranging from 0.08 to $0.03 \%(\mathrm{w} / \mathrm{v})$. To avoid loss of functionality dissolved GelMA was stored in the dark at $4{ }^{\circ} \mathrm{C}$.

\section{$\underline{2.1 .2 \text { Volumetric bioprinting }}$}

For volumetric 3D bioprinting a printer prototype from Readily3D (EPFL Innovation Park, Lausanne, Switzerland) and the updated Tomolite v1.0 printer in combination with the updated Apparite software were used. To optimize printing settings and to achieve clearly defined constructs, laser dose tests were conducted for each GelMA resin. Defined spots were irradiated into a cuvette filled with solidified resin by the inbuild laser $(\lambda=405 \mathrm{~nm})$ for varying exposure times ranging from 2 to 64 seconds and varying average light intensities ranging from 1 to 32 $\mathrm{mW} / \mathrm{cm}^{2}$. The light dose threshold $\left(\mathrm{mJ} / \mathrm{cm}^{2}\right)$ required for precise polymerization, but minimal off-target exposure, was calculated by multiplying exposure time with the average light intensity of the weakly visible polymerized spots in the cuvette. Light doses in the range of 100 to $600 \mathrm{~mJ} / \mathrm{cm}^{2}$ were identified and used for different GelMA solutions depending on the resin batch, photocrosslinker concentration and cell number.

Construct printing was performed in sterile glass vials with $18 \mathrm{~mm}$ or $8 \mathrm{~mm}$ diameter in a volume of $3 \mathrm{ml}$ or $1 \mathrm{ml}$ resin, respectively. For cellular prints $3 \times 10^{6}$ cells $/ \mathrm{ml}$ resin were used. The required number of cells was pelleted by centrifugation after harvesting and directly immersed in prewarmed, liquefied $\left(37^{\circ} \mathrm{C}\right)$ GelMA. After thorough mixing, the cell suspension was rapidly solidified at $4{ }^{\circ} \mathrm{C}$ to prevent cell sedimentation and it was proceeded to printing immediately. The bioink formulation remained solid during the whole printing process $(<60 \mathrm{~s})$ although the bioprinter was stored at ambient temperature $\left(22-23{ }^{\circ} \mathrm{C}\right)$. After printing, the cell suspension was warmed to $37^{\circ} \mathrm{C}$ to liquefy the non-crosslinked resin, the printed construct was washed in warm PBS and transferred to medium for cultivation.

The predetermined refractive index of GelMA (1.37) was applied to acellular and cellular prints and a peak-to average power ratio (PAPR) of 6:1 was chosen to print with optimal resolution at reasonable printing speed. Construct models for volumetric printing were designed as Standard Triangle Language (STL) files using the stereolithography computer-aided design (CAD) software FreeCAD. The STL file of the femur model was provided by Readily3D.

\subsection{3 in-situ Photo-Rheology}

Rheological measurements of bioinks were performed with the rheometer MCR 302 (Anton Paar, Germany), using a parallel plate with $20 \mathrm{~mm}$ diameter. During time sweep measurements with an interval of 6 seconds for 5 minutes, UV-curing of GelMA resins was induced after 60 seconds by illumination with an UV-LED lamp $(\lambda=365 \mathrm{~nm}$, light intensity $35 \%(70 \% \triangleq 20$ 
$\mathrm{mV} / \mathrm{mm}^{2}$ ), Thorlabs). The storage moduli (G') and loss moduli (G') were recorded to assess the crosslinking ability and terminal stiffness of the used resins. $45 \mu 1$ of resin were loaded and the gap was set to $0.1 \mathrm{~mm}$. To prevent drying, wet tissue paper was placed within the temperature chamber $\left(25^{\circ} \mathrm{C}\right)$. Measurements for each resin were triplicated.

\subsubsection{Scaffold mechanics}

To characterize mechanical scaffold properties, the compressive modulus of cellular and acellular printed constructs was determined with a Zwick material testing machine (Zwick 1456, Germany). Unconfined uniaxial compression was tested with a preload of $5 \mathrm{mN}$, and a strain rate of $1 \mathrm{~min}^{-1}$ until a $50 \%$ maximal construct deformation was reached. Ramp compression at a speed ratio of $0.01 \mathrm{~mm} \mathrm{~s}^{-1}$ was applied to obtain stress-strain curves. Disk shaped triplicates (3-6 mm, $\mathrm{h}=1-2 \mathrm{~mm}$ ) were tested at room temperature and the compressive modulus was calculated within the strain range of $5 \%$ to $10 \%$. Measurements of acellular samples was performed after incubation in PBS at $37^{\circ} \mathrm{C}$ for 24 hours and cellular samples were tested after cultivation in cell culture conditions on day 1, 7 and 28 after printing.

\subsection{Cell cultivation}

Cells were maintained at $37^{\circ} \mathrm{C}, 5 \% \mathrm{CO}_{2}$, in $\mathrm{T} 175$ triple flasks and passaged at $80 \%$ confluency using $0.05 \%$ trypsin-EDTA solution (incubation for 5 minutes, $37{ }^{\circ} \mathrm{C}$, gentle tapping to ensure detachment), followed by centrifugation at $300 \mathrm{~g}$ for 10 minutes at $4{ }^{\circ} \mathrm{C}$. All cells were tested negative for mycoplasma contamination using a Universal Mycoplasma Detection Kit (ATCC).

\section{$\underline{\text { Human Mesenchymal Stem Cells (hMSC) }}$}

hMSCs (Normal Human Bone Marrow Derived Mesenchymal Stem Cells, pooled donor, Lonza) were used for experiments in passage 4 to 7 and were cultivated in Dulbecco's Modified Eagle's Medium (DMEM, Gibco) containing 10\% fetal bovine serum (FBS, Gibco) and 1\% antibiotic-antimycotic solution (Penicillin-Streptomycin-Fungizone, Gibco). For cell expansion, medium was supplemented with 1\% MEM non-essential amino acids (Gibco) and $0.001 \%$ basic fibroblast growth factor (bFGF, Gibco, Thermo Fischer Scientific). To induce osteogenic differentiation, medium supplemented with ascorbic acid (50 $\mu \mathrm{g} / \mathrm{ml}$, SigmaAldrich), dexamethasone (100 nM, Sigma-Aldrich) and beta-glycerophosphase (10 mM, Acros Organics, Thermo Fischer Scientific) was used.

\section{$\underline{\text { Human Umbilical Vein Endothelial Cells (HUVEC) }}$}

HUVECs (Human Umbilical Vein Endothelial Cells, pooled donor, Lonza) were used in passage 2 to 6 and were cultivated in Endothelial Cell Growth Medium-2 (EBM-2 medium supplemented with EGM-2 SingleQuots supplements, Lonza). 


\section{Endothelial Co-culture}

hMSCs and HUVECs were harvested separately and mixed in a 5:1 ratio. Fully supplemented osteogenic hMSC medium and EGM-2 medium was mixed in a ratio of 1:1 and used for cultivation of co-cultures.

\subsection{Imaging and Image analysis}

\subsubsection{Microscopy}

$2 \mathrm{D}$ cultures and $3 \mathrm{D}$ constructs were imaged using a confocal laser scanning microscope (Zeiss LSM880) and overview images of acellular constructs were taken with a stereomicroscope (Leica Stereo M205 FA). The respective software ZEN and LAS X were used. Other acellular construct images were captured with a macro lens camera (Xiaomi). When imaged in air, constructs were transferred to a dry surface and surface water was wiped with tissue paper.

Images for viability assessment were quantified using the automated particle analysis plugin on ImageJ (NIH). The 3D rendering software Imaris (Oxford Instruments) was used to visualize z-stacks as 3D-rendered maximum intensity projections.

\subsubsection{Staining of $3 \mathrm{D}$ constructs}

To enhance contrast of 3D constructs, different dyes were used for staining. Gels were incubated for up to 24 hours until sufficiently stained in $10 \%$ alcian blue solution in PBS for brightfield imaging, or in 1\% EosinY dissolved in PBS for fluorescent imaging.

\subsubsection{Microcomputed tomography (microCT)}

Scans with a voxel resolution of $34 \mu \mathrm{m}$ were performed with a micro-CT45 device (Scanco Medical) with the following parameters: energy $=45 \mathrm{kVp}$, current $177 \mu \mathrm{A}$, integration time 600 ms. The micro-computed tomography (micro-CT) images were Gaussian filtered $(\sigma=1.2)$ and the scaffold was distinguished from the background using a threshold of $114 \mathrm{mg}$ hydroxyapatite $/ \mathrm{cm}^{3}$. Component labeling was used to remove structures smaller than 150 connected voxels. 3D images of acellular printed constructs were reconstructed from dry scans in air. ECM mineralization of cellular constructs with mono- and co-cultures (each $n=4$ ) were monitored by weekly micro-CT scans with the same settings for 6 weeks. To avoid scaffold moving, samples were fixed between mesh holders and cultivated in bioreactors with a volume of $5 \mathrm{ml}$. Bioreactors were outside of the incubator during the scanning time of ca. $75 \mathrm{~min}$. 


\subsection{Functional analysis of bioprinted constructs}

\subsubsection{Cell Viability and Morphology}

Cell viability was assessed by performing live/dead assays using the cell-permeant dye CalceinAM (1 mM stock in DMSO, used 1:500 in PBS) and the cell-impermeant dye Ethidium homodimer-1 (2 mM stock in DMSO, used 1:1000 in PBS). 2D and 3D cultures were incubated for 10 or 30 minutes respectively at $37^{\circ} \mathrm{C}$, subsequently washed with medium and imaged within one hour.

Cellular morphology was assessed at different timepoints after fixation with paraformaldehyde (PFA) $(4 \%, 15 / 30 \mathrm{~min}$ for 2D/3D cultures respectively, RT). Unspecific binding sites were blocked for $>1$ hour with $1 \%$ bovine serum albumin (BSA) and cell membranes were permeabilized with $0.2 \%$ Triton-X100 in PBS $(0.1 \%$ BSA) for 10 minutes. Cell nuclei and the actin cytoskeleton were stained with Hoechst (1 mg/ml, 1:1000) and Phalloidin tetramethyl rhodamine (TRITC) $(1: 1000)$ in PBS $(0.1 \%$ BSA) ( $>2$ hours, RT). Samples were kept dark and stored at $4^{\circ} \mathrm{C}$ in PBS.

\subsubsection{Quantification of gene expression}

RNA isolation

Samples for gene expression quantification were collected after cultivation for 1, 7, 14, 21, 28, 35 and 42 days. RNA was directly isolated from $2 \mathrm{D}$ cultures $\left(1 \times 10^{5}\right.$ cells $\left./ 10 \mathrm{~cm}^{2}, \mathrm{n}=3\right)$ following a TRIzol-based protocol. For cell lysis $1 \mathrm{ml}$ TRIzol was added to each sample and incubated for 5 minutes at RT. The cell suspension was transferred into a reaction tube, mixed with 200 $\mu 1$ Chloroform and vortexed for 15 seconds. After 3 minutes samples were centrifuged at full speed (>12000 g) for 15 minutes and the upper water phase containing RNA was aspirated and transferred into a precooled tube. An equal volume of isopropanol was added, and samples were incubated for $10 \mathrm{~min}$ followed by 10 minutes centrifugation. The supernatant was discarded, the precipitate was washed with $80 \%$ ethanol $(\mathrm{v} / \mathrm{v})$ and centrifuged again for 5 minutes. The supernatant was removed, and samples were left open to dry completely for at least 10 minutes. The precipitated RNA was dissolved in $20 \mu 1$ RNase-free water.

Printed 3D constructs for RNA analysis were segmented with a scalpel into smaller pieces immediately after printing $\left(50 \mathrm{~mm}^{3}\right.$ with $3 \times 10^{6}$ cells $\left./ \mathrm{ml}\right)$ and were subsequently treated as independent samples. RNA was isolated from 3D samples $(n=2-3)$ by combining a TRIzolbased cell lysis with a RNeasy Mini Kit (Qiagen). Samples were collected at aforementioned timepoints, snap frozen in liquid nitrogen and stored at $-80^{\circ} \mathrm{C}$ until further usage. 
For RNA isolation $300 \mu \mathrm{l}$ TRIzol were added to the frozen 3D samples and the gels were dissociated in $1.5 \mathrm{ml}$ reaction tubes using a rotating pellet pestle grinder. Afterwards additional $400 \mu 1$ of TRIzol were added, samples were incubated for 5 minutes at RT and centrifuged at full speed (>12000 g) for 5 minutes. To remove gel remnants that could block spin columns the supernatant was transferred to QiaShredder columns (Qiagen) and centrifuged for 2 minutes. $700 \mu \mathrm{l}$ of $70 \%$ ethanol (v/v) was mixed with the flow through and the total volume was transferred to RNeasy spin columns. The following steps were performed according to manufacturer's instructions including DNase I digestion. RNA was eluted in $20 \mu 1$ RNase-free water. The RNA concentration of all samples was measured using a NanoDrop spectrophotometer $(\lambda=260 \mathrm{~nm})$ and samples were stored at $-80^{\circ} \mathrm{C}$ until further usage.

\section{cDNA synthesis and $q P C R$}

$500 \mathrm{ng}$ or $200 \mathrm{ng}$ of isolated RNA from 2D and 3D samples, respectively, were reverse transcribed into cDNA in a volume of $10 \mu 1$ using the PrimeScript RT Master Mix (Perfect Real Time, TaKaRa) $\left(37^{\circ} \mathrm{C}, 15\right.$ minutes; $85^{\circ} \mathrm{C}, 5$ seconds $)$.

20-25 ng cDNA was subsequently used for gene expression analysis via qPCR using TaqMan Fast Universal PCR Master Mix (2x), no AMP erase UNG $\left(95^{\circ} \mathrm{C}, 20\right.$ seconds; 43 cycles of $95^{\circ} \mathrm{C}, 1$ second; $60^{\circ} \mathrm{C}, 20$ seconds) in a CFX96 real-time PCR system (BioRad). Expression levels of two housekeeping genes ( $\beta$-actin (ACTB), glyceraldehyde 3-phosphate dehydrogenase (GAPDH)) were used to normalize expression levels of eight osteogenic marker genes (RUNX2, BGLAP, DMP1, ALPL, PDPN, SOST, COL1a2, MMP14) which were analyzed with specific TaqMan probes (Applied Biosystems, see Table S1).

Obtained data was analyzed for relative gene expression after normalization to two housekeeping genes $(\Delta \mathrm{Ct})$ with the BioRad CFX Maestro software and the analysis software qBase+ (BioGazelle).

\section{ALP/DNA quantification}

Activity levels of alkaline phosphatase (ALP) in relation to DNA expression levels were quantified as marker for osteoblast differentiation after cultivation for 1, 7, 14, 21, 28, 35 and 42 days. ALP activity quantification in the crude lysate of $2 \mathrm{D}\left(1 \times 10^{5}\right.$ cells $\left./ 10 \mathrm{~cm}^{2}, \mathrm{n}=3\right)$ and $3 \mathrm{D}$ (50 $\mathrm{mm}^{3}$ with $3 \times 10^{6}$ cells $/ \mathrm{ml}, \mathrm{n}=2-3$ ) samples was performed according to manufacturer instructions of the Alkaline Phosphatase Assay Kit (colorimetric) (abcam).

2D samples were collected at aforementioned timepoints by adding $100 \mu$ of provided ALP assay buffer to washed $2 \mathrm{D}$ cultures and cells were harvested using a cell scraper. 3D samples 
were directly transferred into $100 \mu \mathrm{l}$ ALP assay buffer. All samples were immediately snap frozen in liquid nitrogen and stored at $-80^{\circ} \mathrm{C}$ until further usage. After thawing, samples were dissociated using a rotating pellet pestle grinder. The ALP assay was performed in duplicates for each sample and standard.

Samples used for the ALP assay were kept at RT for 48 hours, centrifuged for 10 mins ( $>12000$ g) and subsequently, $12.5 \mu \mathrm{l}$ of the supernatant was used for DNA quantification using QuantiT PicoGreen dsDNA Quantification Kit (Invitrogen) following the manufacturer's instructions. Standards containing 0, 10, 50, 250 and 1000 ng DNA/ml were prepared and measured in a total volume of $100 \mu 1$ using a plate reader $(480 \mathrm{~nm} / 520 \mathrm{~nm})$.

To correct for differences in cell number, ALP activity was normalized to the DNA content. For co-cultures, the contribution of HUVECs to the total DNA was subtracted based on the initial co-culture ratio of $5: 1$.

\subsection{Perfusable vascularization model}

$3 \mathrm{D}$ constructs with perfusable channels $(\varnothing<1 \mathrm{~mm})$ were designed and printed in a hMSCcontaining cell suspension $\left(3 \times 10^{6}\right.$ cells $\left./ \mathrm{ml}\right)$. After cultivation for 7 days in osteogenic medium, HUVECs $\left(5 \times 10^{6}\right.$ cells $\left./ \mathrm{ml}\right)$ were suspended in Collagen I gel $(1 \mathrm{mg} / \mathrm{ml}, \mathrm{pH} 7.4)$ and loaded into the perfusable channels using a blunt-tip cannula, $(\varnothing 0.8 \mathrm{~mm})$. To trace HUVECs in co-culture, cells were labelled with the live-cell labelling dye Vybrant DiD (Invitrogen). Therefore, $1 \mathrm{ml}$ cell suspension with $5 \times 10^{6}$ cells $/ \mathrm{ml}$ was mixed with $25 \mu$ l cell-labeling solution $(1 \mathrm{mM})$ and incubated for 30 minutes at $37^{\circ} \mathrm{C}$. The capability of endothelial cells to self-organize was exploited to create an endothelial lining of the channels and constructs were imaged with a confocal microscope 7 days after seeding. Constructs were immersed for 30 minutes in the cellpermeant dye calcein-AM ( $2 \mu \mathrm{M}$ in PBS) before seeding to label hMSCs.

\subsection{Statistical Analysis}

Statistical data analysis was performed using GraphPad Prism 8.2.0. Normality was assumed due to low sample numbers and parametric tests were performed to assess significance. Student's t-test or Analysis of Variance (ANOVA) were applied for pairwise comparison or global comparison between experimental groups, respectively. The Holm-Sidak method was applied to correct for multiple unpaired comparisons using t-tests which assume a similar data scattering among populations at different timepoints. Significance of p-values is defined as $\alpha<$ $0.05(*),<0.01(* *)$ and $<0.001(* * *)$. 


\section{Results and Discussion}

\subsection{Bioink optimization for volumetric bioprinting}

This study explored the use of volumetric 3D bioprinting for generating heterocellular 3D constructs that exhibit enhanced osteogenic differentiation in vitro (Figure 1). The ability of volumetric 3D bioprinter commercialized by Readily3D [6] to rapidly generate 3D cell-laden constructs elucidated its superiority to traditional extrusion-based 3D printers. This supremacy is justified by the very short printing time $(<1 \mathrm{~min})$ for centimeter-scale constructs with micrometer-scale resolution (Supplementary Video S1).

The volumetric bioprinting process is illustrated in Figure S1A. After cell harvesting, hMSCs were mixed with GelMA/LAP ink formulations at $37^{\circ} \mathrm{C}$ in a glass vial. Next, the bioinks were cooled down to $4{ }^{\circ} \mathrm{C}$ for $15 \mathrm{~min}$ to form a physically crosslinked cell-laden gel before bioprinting at ambient temperature. Since the bioprinting process was fast, the ink formulation remained solid. After warming to $37^{\circ} \mathrm{C}$ for $10 \mathrm{~min}$, the printed constructs were obtained and transferred to cell culture media.

A

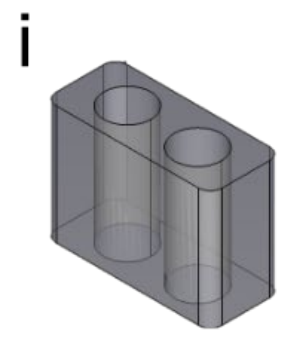

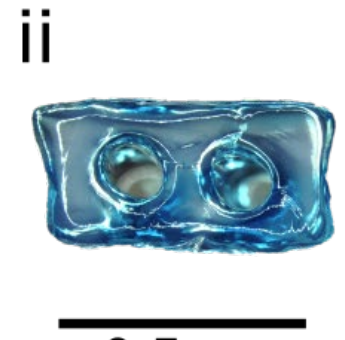

$0.5 \mathrm{~cm}$

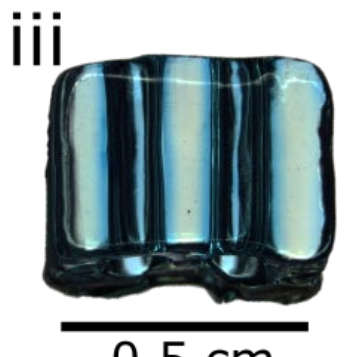

$0.5 \mathrm{~cm}$

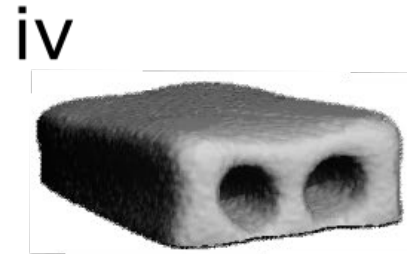

$0.5 \mathrm{~cm}$
B

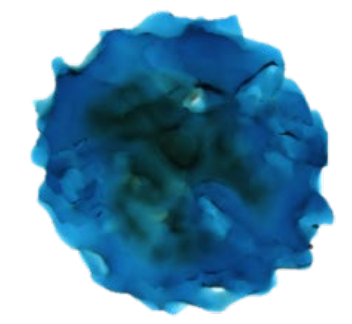

$1 \mathrm{~cm}$
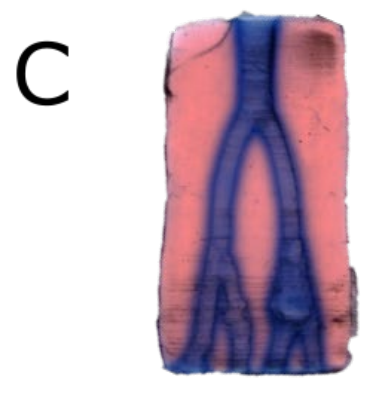

$1 \mathrm{~cm}$
$\mathrm{D}$

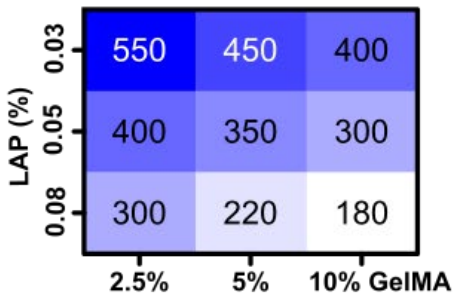

Figure 2: Volumetric bioprinting of in vitro models with hollow and macroporous architecture. A) Volumetric printing of a simplified 3D model with hollow channels: the CAD model (i), top view (ii), side view (iii); and a 3D reconstruction by microCT scannning (iv). Printed samples were stained with Alzian blue to increase contrast. B) Photograph of a printed macroporous femur-like model. Scale bar $=1 \mathrm{~cm}$. C) Photograph of a printed vascular branch model by initial staining with EosinY (pink) and subsequent perfusion of channels with Alzian blue (blue). A bioink with 5\% GelMA and 0.05\% LAP was used for all gel constructs. Scale bar $=1 \mathrm{~cm}$. D) Optimal laser doses for volumetric printing of ink formulations with different GelMA/LAP concentrations. 
Firstly, we sought to characterize and identify optimal bioink compositions that support high level of cell viability, cell growth and structural stability of the hydrogels after bioprinting. The rationale for GelMA as bioink of choice is based on its excellent biocompatibility and its frequent use in 3D cell culture [25-28]. The printing of different centimeter-scale 3D models (Figure 2) with reliable performance was achieved using resins with GelMA concentrations of $2.5 \%, 5 \%$ and $10 \%$ in combination with varying photoinitiator (LAP) concentrations $(0.03 \%$, $0.05 \%, 0.08 \%$ ). Constructs with hollow channels $(\varnothing \leq 1 \mathrm{~mm})$ (Figure 2A, C) were designed and printed with the aim to be adoptable for future perfusion dynamic culture studies. Additionally, a macroporous femur model was successfully printed to resemble the architecture of trabecular bone (Figure 2B). High-resolution 3D reconstructions of the printed models were generated through micro-CT scans with a voxel resolution of $34 \mu \mathrm{m}$, and were used to visually confirm the printing precision. The STL model channel diameter of $1 \mathrm{~mm}$ could be precisely recreated (Figure 2A, iv). We confirm that soft, porous constructs, which traditionally could only be printed with additional supporting structures in multiple steps, can be easily fabricated in single step by volumetric bioprinting within seconds.

To achieve high printing precision, the optimal laser settings have to be determined and validated regularly because the functionalization degree and reactivity of GelMA bioinks show batch variation, and changes of physicochemical properties within one batch with ongoing warming cycles and premature polymerization. To mimic the volumetric printing process, a multi-step rheology measurement of an ink (5\% GelMA, 0.05\% LAP) was performed (Figure S1B). By cooling the sample from $37^{\circ} \mathrm{C}$ to $4{ }^{\circ} \mathrm{C}$ at a rate of $1{ }^{\circ} \mathrm{C} / \mathrm{min}$, both storage $\left(\mathrm{G}^{\prime}\right)$ and loss modulus (G',) was monitored. A sol-gel transition was observed at $16{ }^{\circ} \mathrm{C}$. Subsequently, the sample was exposed to UV-LED $365 \mathrm{~nm}$ irradidation $\left(10 \mathrm{~mW} / \mathrm{cm}^{-2}\right)$ for covalent crosslinking of GelMA via free radical polymerization, which resulted in a rapid increase in shear modulus $\left(\mathrm{G}^{\prime} \sim 3.2 \mathrm{kPa}, \mathrm{G}^{\prime}, \sim 450 \mathrm{~Pa}\right)$ after 5 min irradiation.

Laser dose tests spanning a broad range of average light intensities were used to identify an appropriate light dose that enables highest printing resolution. The required light dose also depends on the power of the inbuilt lasers in the different printer prototypes used in this study, as well as on the diameter of the printing vessel $(8 \mathrm{~mm} / 18 \mathrm{~mm})$. Generally, required light doses are inversely correlated to GelMA and LAP concentration whereby the latter has a greater effect (Figure 2D). The required light dose for printing a bioink with 10\% GelMA and $0.08 \%$ LAP was approximately $180 \mathrm{~mJ} / \mathrm{cm}^{2}$, whereas the dose required for another bioink with $2.5 \%$ 
GelMA, $0.03 \%$ LAP was approximately $550 \mathrm{~mJ} / \mathrm{cm}^{2}$. All other tested GelMA/LAP concentration combinations were printable within this range (Figure 2D). The required light dose directly correlates with the printing duration. However, the printing time does not increase when scaling up the construct volume as long as the same irradiation intensity is supplied [6]. Nevertheless, even light doses above $500 \mathrm{~mJ} / \mathrm{cm}^{2}$ achieved complete polymerization of constructs with a volume of more than $10 \mathrm{~cm}^{3}$ in less than one minute.

Although a broad range of GelMA/LAP concentrations were printable, only a few ink compositions were suited for elaborate experimental procedure requiring manual handling. While Bernal et al. used 10\% GelMA throughout in their study, we reasoned that an optimal mechanical stiffness and matrix permissiveness are essential for cell-matrix remodeling and hMSC differentiation [29-31]. Construct stability mainly depends on GelMA concentration. Bioinks containing 5\% GelMA were chosen for future experiments as a good tradeoff between lower stiffness compared to $10 \%$ GelMA, and improved gel stability compared to $2.5 \%$ GelMA. Furthermore, the printability of a bioink containing 2.5\% GelMA and 2.5\% sacrificial gelatin (total gelatin concentration of 5\%) was confirmed. Aiming for an in situ softening environment, these constructs are expected to exhibit time-dependent decrease of gel stiffness and enlargement of porosity with ongoing incubation and resultant release of gelatin. Interestingly, all GelMA constructs with sacrificial gelatin at varying LAP concentrations showed enhanced ALP expression compared to the control group, implying the improved permissiveness. However, it is important to note that this improvement was accompanied with the compromise on printing precision (Figure S2). With this information in mind, 5\% GelMA without sacrificial gelatin was determined as the optimal resin for further experiments since it combines high printing precision, structural fidelity, and ease of handling. Additionally, cellular constructs with 5\% GelMA showed enhanced osteogenic differentiation reflected by the increased relative gene expression of the osteocytic marker gene PDPN compared to constructs with a GelMA concentration of $10 \%$ (Figure $\mathbf{S 3}$ ).

\subsection{Effect of photoinitiator concentration}

We investigated whether the LAP concentration in combination with the respective light dose influences printing precision and cell-compatibility. Construct staining with the freely diffusible, fluorescent dye Eosin Y allowed confocal microscopic imaging at high resolution (Figure 3A). From a side view, linear structures could be observed as printing artifacts at any concentration but no differences in printing precision from neither side (Figure 3B, i-iii) nor top view (Figure 3B, iv-vi) could be observed. This observation suggests that GelMA-based 
bioinks have excellent printability provided the light dose and photoinitiator concentrations are carefully selected.

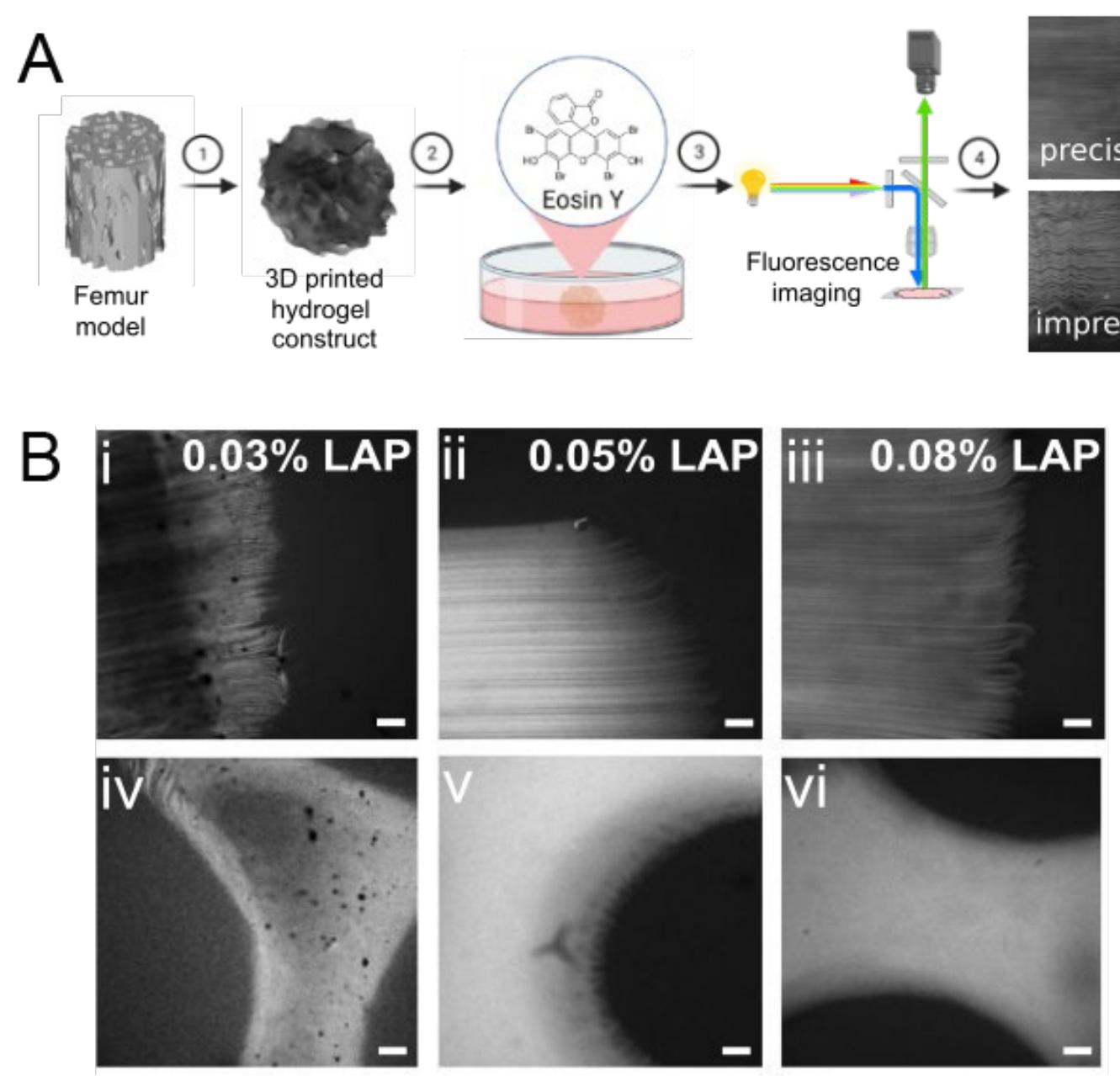

Figure 3: Assessment of the printing precision by Eosin $Y$ staining and confocal fluorescence microscopy. A) Schematic workflow of the precision assessment in volumetrically printed 3D constructs. Printed constructs were incubated in EosinY solution for staining and then imaged with confocal laser scanning microscopy. Top and side view images revealed printing artifacts and precision (printed matrices in grey). Created with BioRender.com. B) Confocal fluorescence microscopy images after Eosin $\mathrm{Y}$ staining of volumetrically printed constructs revealing the structural fidelity of printed constructs as a function of LAP concentration (side view: i, ii, iii; top view: iv, v, vi), scale bars $=100$ $\mu \mathrm{m}$.

Thereafter, we investigated the physical properties of hydrogel constructs by in situ photorheology and Zwick mechanical characterization to test if matrix stiffness affects cell spreading (Figure 4 A-C). The crosslinking efficiency of the bioinks were assessed by time-lapsed in situ photo-rheology with UV-365nm irradiation (Figure 4A-B). The evolution of storage $\left(\mathrm{G}^{\prime}\right)$ and loss (G") moduli was monitored at an interval of $10 \mathrm{~s}$. All compositions reached a G'-plateau in the range of $850 \mathrm{~Pa}-1850 \mathrm{~Pa}$ (Table 1) after an irradiation for $4 \mathrm{~min}$. No significant differences in the $\mathrm{G}^{\prime}$-plateau moduli were observed between the $0.03 \%$ and $0.05 \%$ LAP groups. However, the gels with $0.08 \%$ LAP exhibited the highest stiffness. Moreover, the sample with $0.03 \%$ LAP 
reached the onset $\left(\mathrm{G}^{\prime}>5 \mathrm{~Pa}\right)$ at ca.116 s, whereas the onset time for the other two groups was

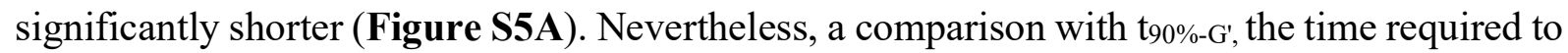
reach $90 \%$ of the G'-plateau value, showed no significant difference (Figure S5B). Together, the $0.03 \%$ LAP group has the lowest photoreactivity, whereas higher LAP concentrations accelerated the photocrosslinking.
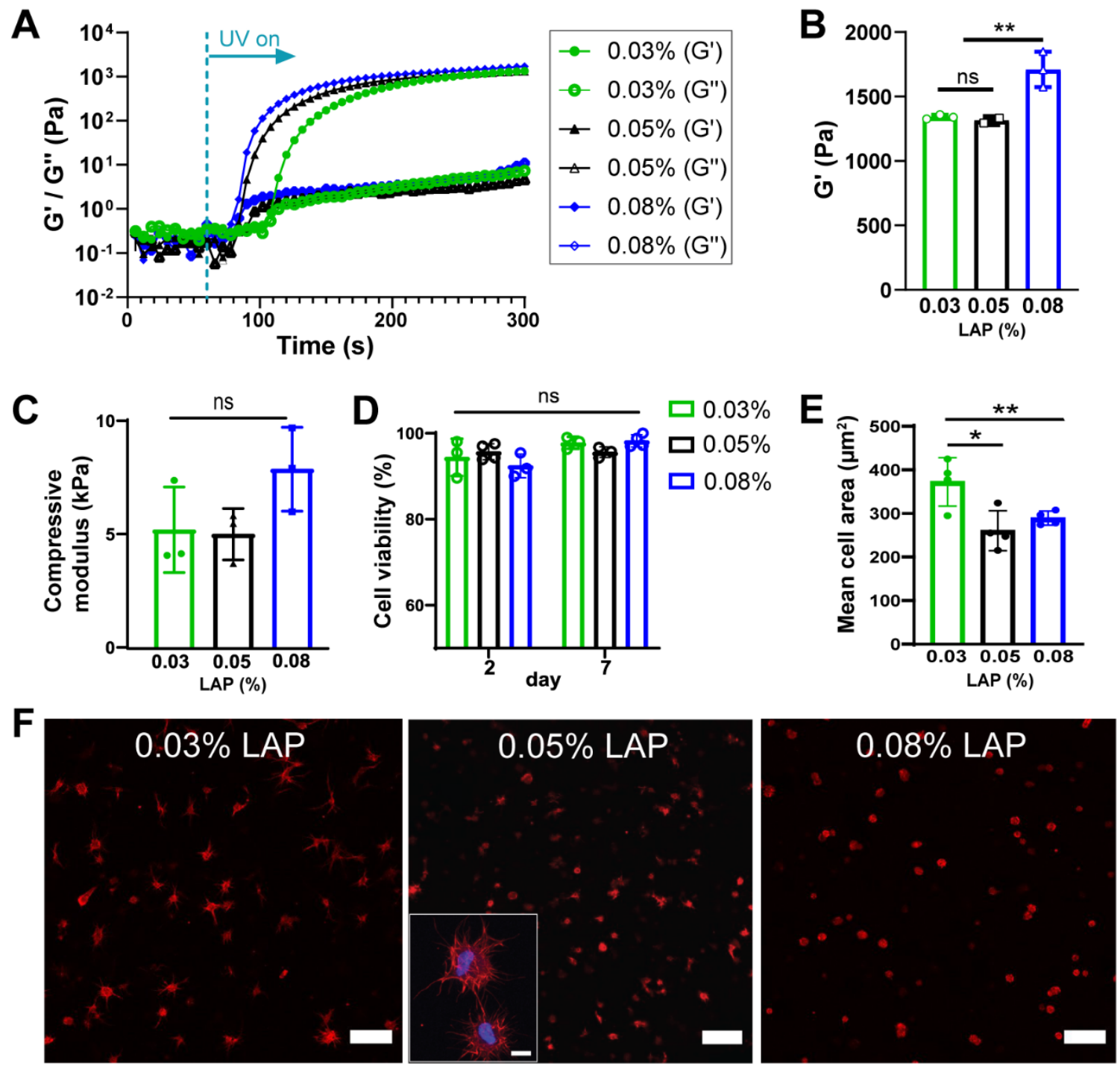

Figure 4: Effects of LAP photoinitiator concentration on gel crosslinking, stiffness, and cell activity. All experiments were performed with inks containing 5\% GelMA and different LAP concentrations $(0.03 \%$, green; $0.05 \%$, black; $0.08 \%$, blue) A) Time sweep of storage and loss moduli (G'/G') of acellular inks with UV-365nm light after 60s (dashed line) $(\mathrm{n}=3)$. Data points represent the mean, error bars are not shown for better visibility. B) Effect of LAP concentration on G'-plateau values determined by photo-rheology. C) Mechanical characterization of acellular printed gel constructs after incubation in PBS for 24h (n=3). D) Viability of cells in printed constructs on day 2 and $7(n=3-4)$. E) Average surface area of embedded cells on day 7 as indicator for cell spreading and dendrite formation. F) Cell morphology in printed constructs at day 7, visualized by actin staining, maximum intensity projection (MIP), scale bars $=100 \mu \mathrm{m}$. Inside image shows cell spreading in the $0.05 \%$ group by actinnuclei staining (red-blue), MIP, scale bar $=20 \mu \mathrm{m}$. Columns and error bars represent mean and standard deviation (B-E), symbols represent data points; $n s \hat{=}>0.05,{ }^{*} \mathrm{p}<0.05,{ }^{* *} \mathrm{p}<0.01,{ }^{* * *} \mathrm{p}<0.001$; oneway ANOVA was performed to compare groups. 
Table 1. Effect of LAP concentration on the photocrosslinking of GelMA. Results determined by in situ photo-rheology: 5\% GelMA, UV-365 $\mathrm{nm}$ at $20 \mathrm{~mW} / \mathrm{cm}^{-2}, 4 \mathrm{~min}$.

\begin{tabular}{|c|c|c|c|c|c|}
\hline LAP (\%) & $\begin{array}{l}\text { G'-plateau }^{\text {a }} \\
\text { (Pa) }\end{array}$ & $\begin{array}{l}\text { G'-plateau }^{\mathrm{a}} \\
\text { (Pa) }\end{array}$ & $\begin{array}{l}\text { loss factor } \\
\left(\times 10^{-3}\right)\end{array}$ & $\begin{array}{l}\mathbf{t}_{\text {onset }} \\
\text { (s) }\end{array}$ & $\begin{array}{l}\mathbf{t}_{90 \%-\mathrm{G}^{\prime}} \\
\text { (s) }\end{array}$ \\
\hline $0.03 \%$ & $1341.6 \pm 16.2$ & $7.4 \pm 3.0$ & $5.5 \pm 2.3$ & $116 \pm 3.5$ & $276 \pm 0$ \\
\hline $0.05 \%$ & $1155.8 \pm 273.9$ & $4.7 \pm 3.4$ & $3.9 \pm 2.2$ & $96 \pm 10.4$ & $264 \pm 6.0$ \\
\hline $0.08 \%$ & $1709.9 \pm 137.8$ & $18.1 \pm 8.6$ & $10.4 \pm 4.1$ & $90 \pm 0$ & $274 \pm 13.9$ \\
\hline
\end{tabular}

${ }^{a} \mathrm{G}^{\prime}, \mathrm{G}^{\prime \prime}$ and loss factor are the plateau values at $300 \mathrm{~s}$;

${ }^{b} t_{\text {onset }}$ is defined at the first data point where $G^{\prime}(t)>5 \mathrm{~Pa}$, indicating the time needed for curing.

c $t_{90 \%-G^{\prime}}$ is defined at the time point where $G^{\prime}(t)$ reached $90 \%$ of the G' values at $300 \mathrm{~s}$.

Additionally, the compressive moduli of 3D printed acellular GelMA constructs after swelling was determined with a Zwick mechanical testing machine (Figure 4C). Stiffness differences between the scaffolds with the two lower $(0.03 \%$ and $0.05 \% \mathrm{LAP} \triangleq 5 \mathrm{kPa})$ and the one higher $(0.08 \%$ LAP $\triangleq 8 \mathrm{kPa})$ LAP concentration were observed, although not being statistically significant. These results are consistent with the previously reported observations of faster crosslinking due to higher number of free radicals [24].

Next, we varied LAP concentrations to investigate the influence of LAP and the respective required light dose on cell-compatibility and permissiveness of printed hydrogel environments with cell viability and morphology assays. A high cell viability of $>92 \%$ after 7 days was reached for all constructs without differences (Figure 4D, Figure S4). However, remarkable differences were observed when assessing the morphology of embedded cells after an actinnuclei staining. Cell spreading with fine dendritic processes could be observed after 7 days for the samples with $0.03 \%$ and $0.05 \%$ LAP concentration (Figure 4F; Supplementary Video S2), whereas cells remained round in $0.08 \%$ LAP containing gels. This suggests that higher LAP concentrations, and the thereby increased exposure of cells to free radicals might pose toxic effects to the cell fidelity without affecting viability directly [32, 33]. The observation of increased cell spreading was confirmed by an increased cell surface area in constructs with lower LAP concentration (Figure 4E). These findings explain the observed delayed osteogenic differentiation of fully embedded cells compared to top-seeded cells on acellular constructs or 2D cultures $[9,18]$. Therefore, $5 \%$ GelMA with $0.05 \%$ LAP was identified as bioink of choice with optimal handling and permissiveness and was used for the following cell culture and functional analysis studies. 


\subsection{Enhancing hMSC osteogenesis with endothelial co-cultures}

Next, we evaluated whether endothelial co-cultures enhanced in vitro osteogenic differentiation and functionality in the 3D printed constructs. Therefore, we assessed marker gene expression by qPCR, performed enzyme activity assays and followed the level of matrix secretion by mechanical testing. A co-culture system was established using stem cells in the osteogenic lineage and endothelial cells (HUVEC), which have the capacity to self-organize and establish intercellular connections. The suitability of the used co-culture medium for both cell types was confirmed by a high viability of $>97 \%$ and unaltered morphology after 7 days (Figure S6).
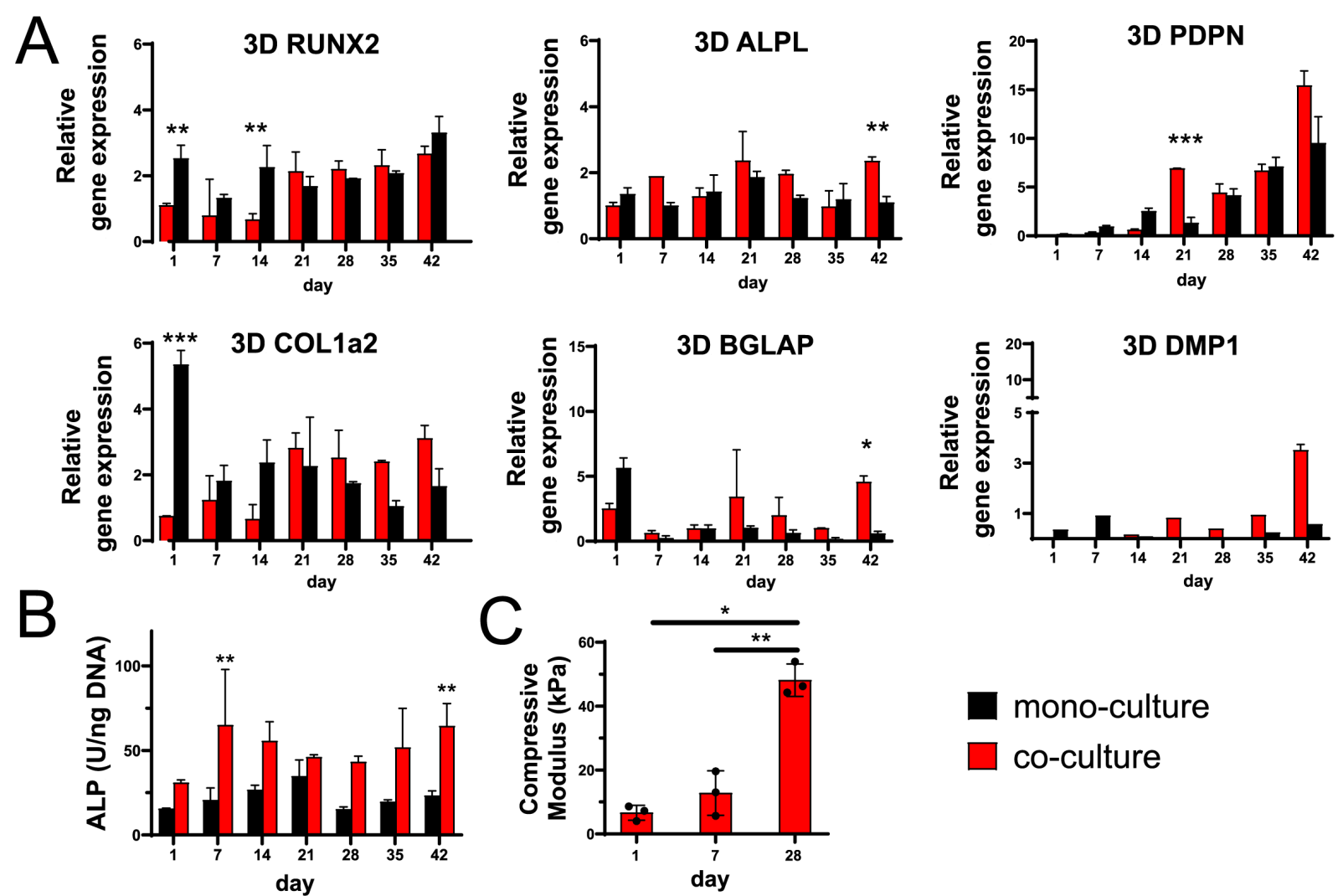

Figure 5: Functional analysis of osteogenic differentiation in co- and mono-cultures in 3D. A) Gene expression analysis of osteoblastic (RUNX2, COL1a2, ALPL, BGLAP) and osteocytic (PDPN, DMP1) marker genes over 6 weeks $(n=2-3)$. Graphs compare mono- with co-cultures in 3D printed cellular constructs. B) ALP activity in osteogenic cultures was normalized to the DNA content $(n=2-3)$. C) Compressive moduli of co-cultured samples after 1,7 and 28 days of cultivation $(n=3)$. Columns and error bars represent mean and standard deviation, symbols represent data points (D); ${ }^{*} \mathrm{p}<0.05$, ${ }^{* *} \mathrm{p}<0.01,{ }^{* * *} \mathrm{p}<0.001 ;$ t-tests with Holm-Sidak correction were used.

Relative gene expression of the osteoblastic and osteocytic markers (ALPL, RUNX2, BGLAP, COL1a2, PDPN, DMP1, SOST) in mono- and co-cultures (i.e., hMSC and hMSC/HUVEC) were quantified by qPCR weekly (Figure 5; Table S1). Mono-cultures of HUVECs were used as the control. Importantly, neither considerable amounts of ALP enzyme activity, nor reasonable expression of any of the relevant osteogenic genes could be observed in HUVEC mono-cultures. Therefore, the contribution of HUVECs to total osteogenic gene expression 
levels in co-cultures was neglected (Figure S7B). Generally, all osteoblastic marker genes were expressed throughout the cultivation time, indicating ongoing osteogenic differentiation.

At the early stage of osteogenesis, hMSCs showed a higher expression of several osteogenic markers in the mono-cultures. The transcription factor RUNX2 is a key marker for the preosteoblast to osteoblast transition. As shown in Figure 5A, RUNX2 expression appeared to be significantly higher in mono-cultures than co-cultures at the early osteogenic stage on day 1 and 14. From 21 days on, its expression in co-culture reached a similar level to mono-culture. A similar trend was observed in the early-stage expression of COLla2 genes, indicating the secretion of Collagen-I as a matrix protein by hMSC-derived bone cells. After 21 days, there was a significant increase of COL1a2 expression in the co-cultures compared to mono-cultures. The ALPL encodes for ALP which is synthesized and secreted by osteoblasts for matrix mineralization. On day 42 a significantly higher expression of $A L P L$ was found in co-cultures.

The gene BGLAP codes for osteocalcin, a bone derived hormone that is solely secreted by osteoblasts. Although on day 1 it was higher expressed in mono-cultures, after 21 days the level of expression significantly increased in the co-cultures (Figure 5A). MMP14, also known as MT1-MMP, is a proteolytic enzyme involved in matrix remodeling during osteogenesis and is predominantly active in mature osteoblasts [34]. It was continuously highly expressed and no significant differences between co- and mono-cultures could be observed (Figure S7A). Together, at the early stage of 3D osteogenesis most osteoblastic marker genes showed a higher level of expression in mono-cultures and then a trend of enhanced gene expression in cocultures at later timepoints were identified. These findings imply the temporal dynamics of gene expression in 3D osteogenesis as well as the significant role of juxta- and/or paracrine signaling in cell activity.

In accordance with this trend, marker genes of early osteocytes were increasingly expressed in $3 \mathrm{D}$ co-cultures. The early osteocytic marker gene PDPN codes for a transmembrane glycoprotein which is important for dendrite elongation. After day 21, it was consistently higher expressed in $3 \mathrm{D}$ co-cultures compared to mono-cultures with the highest reached expression levels after 42 days. Moreover, the early osteocytic marker DMP1, a regulator for matrix mineralization, was higher or exclusively expressed in co-cultures compared to mono-cultures, especially in a later stage after day 21 . No expression of the marker gene SOST for mature osteocytes was observed at any time point. Similar to the effects observed on the expression of osteoblast markers, co-cultures tend to the enhance expression of early osteocytic marker genes. 
To confirm the ALPL gene expression, an ALP activity assay was performed (Figure 5B, Figure S8B). HUVEC controls showed no considerable ALP activity which allowed direct comparison between 2D/3D mono- and co-cultures (Figure S7A). ALP activity in 3D was not matched to the $A L P L$ gene expression patterns and rather stable expressed in both, mono- and co-culture, during the whole observation time. ALP activity in co-cultures was generally increased compared to mono-cultures with significant differences on day 7 and day 42 .

The differences in gene expression and enzyme activity between mono- and co-cultures we also monitored in 2D cultures, additionally to the characterization of 3D printed constructs (Figure S8). Interestingly, we observed the effects of co-cultures on osteogenic differentiation being less prominent in $2 \mathrm{D}$ cultures compared to $3 \mathrm{D}$. This observation can be explained by the potential of $3 \mathrm{D}$ environments to allow endothelial self-organization into vasculature-like structures, and related alterations in signaling [35]. However, further validation by cell tracing, cell tracking and molecular analysis is required to prove this hypothesis. Presence of a selforganized vasculature-like network would promote cell-cell communication, nutrient transport and tissue maturation for a long-term functional 3D culture, even though top-seeding approaches onto porous scaffolds are still frequently used [9]. Additionally, it has to be noted that a direct comparison of $2 \mathrm{D}$ and $3 \mathrm{D}$ cultures in this study can only be done with a certain reluctance since cell number, spatial organization, culturing environment and RNA isolation techniques differed significantly.

Gene expression analysis and enzymatic assays are powerful tools to assess construct functionality. But especially for bone tissue formation, the level of matrix mineralization is an important indicator. To evaluate this, we followed matrix mineral deposition by time-lapsed micro-CT scans. Printed mono- and co-cultures were cultivated in bioreactors and scanned weekly for 6 weeks, but no sufficient mineralization could be visualized at any time point. Thus, we investigated whether other matrix parameters such as the gel mechanics changed over time. Changes in the compressive moduli of $3 \mathrm{D}$ co-culture samples were monitored by mechanical testing on day 1, 7 and 28 after printing. Here, the compressive moduli of the gel constructs increased from about $6 \mathrm{kPa}(\mathrm{d} 1)$ to $13 \mathrm{kPa}(\mathrm{d} 7)$ and $46 \mathrm{kPa}(\mathrm{d} 28)$, with an 8-fold increase between day 1 and 28 (Figure 5C). Moreover, an increasing level of gel turbidity was observed during 3D co-culture (Figure S9). These results imply that there has been remarkable level of matrix secretion during 3D co-culture. Nevertheless, future work is warranted to investigate if there are remarkable differences in mineral formation between co-culture and mono-culture and to devise strategies to promote tissue maturation with a higher level of mineralization. 
bioRxiv preprint doi: https://doi.org/10.1101/2021.11.14.468504; this version posted November 29, 2021. The copyright holder for this preprint (which was not certified by peer review) is the author/funder. All rights reserved. No reuse allowed without permission.

\subsection{Volumetric bioprinting of pre-vascularized bone constructs}

Given the promise of our 3D bioprinted co-culture platform, we further explored volumetric printing of a perfusable pre-vascularized bone construct. Using a 5\% GelMA bioink with $0.05 \%$ LAP, single hMSCs were printed inside 3D constructs with a hollow channel and predifferentiated into the osteogenic lineage by cultivation in osteogenic medium (Figure 6A). On day 7, bottom-closed channels were loaded with a dense HUVEC cell suspension in a supporting Collagen-I hydrogel, which provides a 3D environment for self-organization of HUVEC cells.

After initial cell seeding, perfusion of channels was restored by removal of covering hydrogel plugs. Confocal 3D imaging after additional 7 days of cultivation confirmed the lining of channels with a self-organized endothelial monolayer (red) (Figure 6B-C; Supplementary Video S3). This pre-vascularized channel model could also be used together with millifluidic perfusion systems to study how flow-induced shear stress promotes cell maturation and matrix mineralization [36]. Our prototype paves the way for adaptor-free perfusion and can eventually drive the development of personalized transplants. However, the resolution of volumetric bioprinting and the endothelial cell seeding methods have to be optimized to reach physiological microvascularization [37].
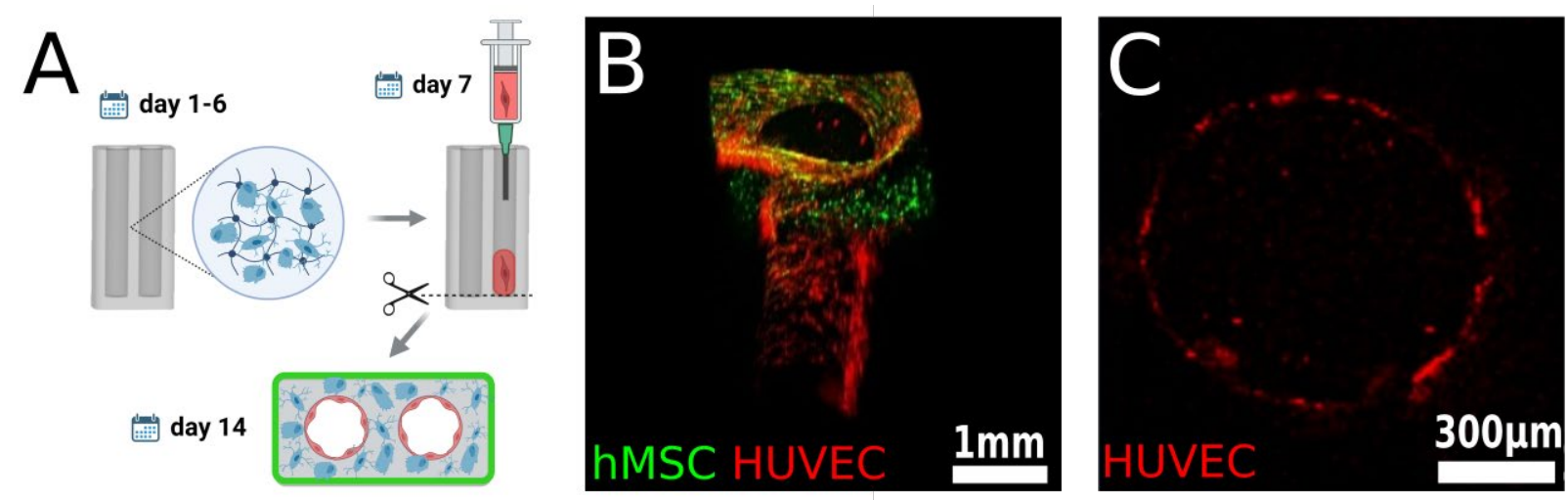

Figure 6: Establishment of heterocellular perfusable pre-vascularization model. A) Schematic experimental procedure of endothelial channel lining in osteogenic 3D constructs: pre-differentiation of hMSC-containing gel constructs for 7 days, injection of endothelial cells with a supporting collagen matrix into the channels, removal of hydrogel plugs to restore medium perfusion, self-organization of endothelial cells into channel lining after 14 days. Created with BioRender.com. B) 3D rendered confocal image of an endothelium-lined channel on day 14. hMSCs on the construct surface were stained with calcein-AM (green), while HUVECs inside the channel were stained with DiD (red), respectively. Scale bar $=1 \mathrm{~mm}$. C) Cross-section confocal image of an endothelium-lined channel on day 14 . HUVECs stained with DiD (red). Scale bar $=300 \mu \mathrm{m}$. 


\section{Conclusion \& Outlook}

In conclusion, we demonstrated ultrafast volumetric tomographic 3D bioprinting of cm-scale cell-laden hydrogel constructs for enhanced in vitro osteogenesis through 3D endothelial coculture. A systematic screening of bioink compositions such as polymer and initiator concentration identified an optimal bioink (5\% GelMA, 0.05\% LAP) that combines good printability and cell-compatibility for tomographic photopatterning. Compared to previously reported bioink using 10\% GelMA, our bioink offers better permissiveness for cell-matrix remodeling and cell-cell communication in 3D. Moreover, we performed functional analysis of hMSC/HUVEC 3D co-cultures and hMSC mono-cultures for six weeks. The results revealed enhanced expression of early osteocytic markers (PDPN and DMP1) in the 3D bioprinted constructs under co-culture after 3 weeks, implying the accelerated osteogenic differentiation by up-regulated juxta- and paracrine signaling in the heterocellular $3 \mathrm{D}$ co-culture system. Additionally, a prototype for a pre-vascularized construct with endothelial cell lining was successfully established. To the best of our knowledge, this is the first study to rapidly fabricate perfusable pre-vascularized bone-like constructs by volumetric $3 \mathrm{D}$ bioprinting.

Nevertheless, the limited level of marix mineralization in combination with the absence of mature osteocytic gene signatures (SOST) emphasize the need to further improve our strategies for in vitro osteocytic differentiation. Similar studies have identified additional parameters that can further drive osteogenesis, such as mechanical stimulation which is a crucial cue for matrix mineralization. The described in vitro platform can be upgraded with mechanical loading experiments to enhance in vitro osteogenesis towards higher level of functionality by employing spinning or dynamic compression bioreactors. In future, we envisage that researchers can greatly take advantages of this co-culture volumetric bioprinting platform for scaled fabrication of more complex human tissues within seconds for applications in regenerative medicine and in vitro drug discovery.

\section{Acknowledgements}

The authors would like to thank Leana Bissig for experimental support with bio-imaging, Dr. Gian Schädli for technical support with micro-CT imaging and Christian Gehre for assistance with qPCR. J.G. acknowledges the financial support from the Heyning-Roelli Foundation. W. Qiu gratefully acknowledges the financial support from the China Scholarship Council (CSC). X.H.Q. is grateful to the financial support by the Swiss National Science Foundation (Project No. 190345). 
bioRxiv preprint doi: https://doi.org/10.1101/2021.11.14.468504; this version posted November 29, 2021. The copyright holder for this preprint (which was not certified by peer review) is the author/funder. All rights reserved. No reuse allowed without permission.

\section{Author Contributions}

J.G. and X.H.Q. conceived the study and designed the experiments, J.G. performed most of the experiments including 3D bioprinting, mono- and co-cultures, confocal laser microscopy imaging and molecular biology assays. W.Q. performed mechanical testing of the hydrogels and analyzed the data. All authors analyzed the results and participated in the manuscript writing, X.H.Q. and R.M. jointly supervised this research.

\section{References}

1. Moroni, L., J.A. Burdick, C. Highley, S.J. Lee, Y. Morimoto, S. Takeuchi, and J.J. Yoo, Biofabrication strategies for $3 D$ in vitro models and regenerative medicine. Nat Rev Mater, 2018. 3(5): p. 21-37.

2. Kim, J., B.K. Koo, and J.A. Knoblich, Human organoids: model systems for human biology and medicine. Nat Rev Mol Cell Biol, 2020. 21(10): p. 571-584.

3. Arslan-Yildiz, A., R. El Assal, P. Chen, S. Guven, F. Inci, and U. Demirci, Towards artificial tissue models: past, present, and future of $3 D$ bioprinting. Biofabrication, 2016. 8(1): $\mathrm{p}$. 014103.

4. Mandrycky, C., Z. Wang, K. Kim, and D.H. Kim, 3D bioprinting for engineering complex tissues. Biotechnol Adv, 2016. 34(4): p. 422-434.

5. Koons, G.L., M. Diba, and A.G. Mikos, Materials design for bone-tissue engineering. Nature Reviews Materials, 2020. 5(8): p. 584-603.

6. Bernal, P.N., P. Delrot, D. Loterie, Y. Li, J. Malda, C. Moser, and R. Levato, Volumetric Bioprinting of Complex Living-Tissue Constructs within Seconds. Adv Mater, 2019. 31(42): p. e1904209.

7. Kelly, B.E., I. Bhattacharya, H. Heidari, M. Shusteff, C.M. Spadaccini, and H.K. Taylor, Volumetric additive manufacturing via tomographic reconstruction. Science, 2019. 363(6431): p. 1075-1079.

8. Rizzo, R., D. Ruetsche, H. Liu, and M. Zenobi-Wong, Optimized Photoclick (Bio)Resins for Fast Volumetric Bioprinting. Advanced Materials, 2021: p. 2102900.

9. Akiva, A., J. Melke, S. Ansari, N. Liv, R. van der Meijden, M. van Erp, F. Zhao, M. Stout, W.H. Nijhuis, C. de Heus, C. Muñiz Ortera, J. Fermie, J. Klumperman, K. Ito, N. Sommerdijk, and S. Hofmann, An Organoid for Woven Bone. Advanced Functional Materials, 2021. 31(17): p. 2010524.

10. Aziz, A.H. and S.J. Bryant, A comparison of human mesenchymal stem cell osteogenesis in poly(ethylene glycol) hydrogels as a function of MMP-sensitive crosslinker and crosslink density in chemically defined medium. Biotechnol Bioeng, 2019. 116(6): p. 1523-1536.

11. Heino, T.J. and T.A. Hentunen, Differentiation of osteoblasts and osteocytes from mesenchymal stem cells. Curr Stem Cell Res Ther, 2008. 3(2): p. 131-45.

12. Guan, M., W. Yao, R. Liu, K.S. Lam, J. Nolta, J. Jia, B. Panganiban, L. Meng, P. Zhou, M. Shahnazari, R.O. Ritchie, and N.E. Lane, Directing mesenchymal stem cells to bone to augment bone formation and increase bone mass. Nat Med, 2012. 18(3): p. 456-62.

13. Assis-Ribas, T., M.F. Forni, S.M.B. Winnischofer, M.C. Sogayar, and M. Trombetta-Lima, Extracellular matrix dynamics during mesenchymal stem cells differentiation. Dev Biol, 2018. 437(2): p. 63-74.

14. Klein-Nulend, J. and L.F. Bonewald, The osteocyte, in Principles of Bone Biology. 2020. p. 133-162.

15. Zhang, C., A.D. Bakker, J. Klein-Nulend, and N. Bravenboer, Studies on Osteocytes in Their $3 D$ Native Matrix Versus 2D In Vitro Models. Curr Osteoporos Rep, 2019. 17(4): p. 207-216.

16. Zhang, J., H. Eyisoylu, X.H. Qin, M. Rubert, and R. Muller, 3D bioprinting of graphene oxide-incorporated cell-laden bone mimicking scaffolds for promoting scaffold fidelity, osteogenic differentiation and mineralization. Acta Biomater, 2021. 121: p. 637-652. 
bioRxiv preprint doi: https://doi.org/10.1101/2021.11.14.468504; this version posted November 29, 2021. The copyright holder for this preprint (which was not certified by peer review) is the author/funder. All rights reserved. No reuse allowed without permission.

17. Zhang, J., E. Wehrle, P. Adamek, G.R. Paul, X.-H. Qin, M. Rubert, and R. Müller, Optimization of mechanical stiffness and cell density of $3 D$ bioprinted cell-laden scaffolds improves extracellular matrix mineralization and cellular organization for bone tissue engineering. Acta Biomaterialia, 2020. 114: p. 307-322.

18. Persson, M., P.P. Lehenkari, L. Berglin, S. Turunen, M.A.J. Finnila, J. Risteli, M. Skrifvars, and J. Tuukkanen, Osteogenic Differentiation of Human Mesenchymal Stem cells in a $3 D$ Woven Scaffold. Sci Rep, 2018. 8(1): p. 10457.

19. $\mathrm{Hu}, \mathrm{K}$. and B.R. Olsen, The roles of vascular endothelial growth factor in bone repair and regeneration. Bone, 2016. 91: p. 30-8.

20. Sasaki, J.-I., M. Hashimoto, S. Yamaguchi, Y. Itoh, I. Yoshimoto, T. Matsumoto, and S. Imazato, Fabrication of Biomimetic Bone Tissue Using Mesenchymal Stem Cell-Derived Three-Dimensional Constructs Incorporating Endothelial Cells. PLOS ONE, 2015. 10(6): p. e0129266.

21. Saleh, F.A., M. Whyte, and P.G. Genever, Effects of endothelial cells on human mesenchymal stem cell activity in a three-dimensional in vitro model. Eur Cell Mater, 2011. 22: p. 242-57; discussion 257.

22. Inomata, K. and M. Honda, Co-Culture of Osteoblasts and Endothelial Cells on a Microfiber Scaffold to Construct Bone-Like Tissue with Vascular Networks. Materials (Basel), 2019. 12(18).

23. Heo, D.N., B. Ayan, M. Dey, D. Banerjee, H. Wee, G.S. Lewis, and I.T. Ozbolat, Aspirationassisted bioprinting of co-cultured osteogenic spheroids for bone tissue engineering. Biofabrication, 2020.

24. Loessner, D., C. Meinert, E. Kaemmerer, L.C. Martine, K. Yue, P.A. Levett, T.J. Klein, F.P. Melchels, A. Khademhosseini, and D.W. Hutmacher, Functionalization, preparation and use of cell-laden gelatin methacryloyl-based hydrogels as modular tissue culture platforms. Nat Protoc, 2016. 11(4): p. 727-46.

25. Yue, K., G. Trujillo-de Santiago, M.M. Alvarez, A. Tamayol, N. Annabi, and A. Khademhosseini, Synthesis, properties, and biomedical applications of gelatin methacryloyl (GelMA) hydrogels. Biomaterials, 2015. 73: p. 254-71.

26. Wang, X., X.-H. Qin, C. Hu, A. Terzopoulou, X.-Z. Chen, T.-Y. Huang, K. Maniura-Weber, S. Pané, and B.J. Nelson, 3D Printed Enzymatically Biodegradable Soft Helical Microswimmers. Advanced Functional Materials, 2018. 28(45).

27. Ovsianikov, A., S. Mühleder, J. Torgersen, Z. Li, X.-H. Qin, S. Van Vlierberghe, P. Dubruel, W. Holnthoner, H. Redl, and R. Liska, Laser photofabrication of cell-containing hydrogel constructs. Langmuir, 2013. 30(13): p. 3787-3794.

28. Qin, X.-H., A. Ovsianikov, J. Stampfl, and R. Liska, Additive manufacturing of photosensitive hydrogels for tissue engineering applications. BioNanoMaterials, 2014. 15(3-4): p. 49-70.

29. Zhang, J., E. Wehrle, P. Adamek, G.R. Paul, X.H. Qin, M. Rubert, and R. Muller, Optimization of mechanical stiffness and cell density of $3 D$ bioprinted cell-laden scaffolds improves extracellular matrix mineralization and cellular organization for bone tissue engineering. Acta Biomater, 2020. 114: p. 307-322.

30. Mc Garrigle, M.J., C.A. Mullen, M.G. Haugh, M.C. Voisin, and L.M. McNamara, Osteocyte differentiation and the formation of an interconnected cellular network in vitro. Eur Cell Mater, 2016. 31: p. 323-40.

31. Murphy, C.M., A. Matsiko, M.G. Haugh, J.P. Gleeson, and F.J. O’Brien, Mesenchymal stem cell fate is regulated by the composition and mechanical properties of collagenglycosaminoglycan scaffolds. Journal of the Mechanical Behavior of Biomedical Materials, 2012. 11: p. 53-62.

32. Ruskowitz, E.R. and C.A. DeForest, Proteome-wide Analysis of Cellular Response to Ultraviolet Light for Biomaterial Synthesis and Modification. ACS Biomater Sci Eng, 2019. 5(5): p. 2111-2116.

33. Bryant, S.J., C.R. Nuttelman, and K.S. Anseth, Cytocompatibility of UV and visible light photoinitiating systems on cultured NIH/3T3 fibroblasts in vitro. J Biomater Sci Polym Ed, 2000. 11(5): p. 439-57. 
34. Manduca, P., A. Castagnino, D. Lombardini, S. Marchisio, S. Soldano, V. Ulivi, S. Zanotti, C. Garbi, N. Ferrari, and D. Palmieri, Role of MT1-MMP in the osteogenic differentiation. Bone, 2009. 44(2): p. 251-65.

35. Andrique, L., G. Recher, K. Alessandri, N. Pujol, M. Feyeux, P. Bon, L. Cognet, P. Nassoy, and A. Bikfalvi, A model of guided cell self-organization for rapid and spontaneous formation of functional vessels. Sci Adv, 2019. 5(6): p. eaau6562.

36. Moser, C., K. Bardsley, A.J. El Haj, M. Alini, M.J. Stoddart, and J.J. Bara, A Perfusion Culture System for Assessing Bone Marrow Stromal Cell Differentiation on PLGA Scaffolds for Bone Repair. Front Bioeng Biotechnol, 2018. 6: p. 161.

37. Mastrullo, V., W. Cathery, E. Velliou, P. Madeddu, and P. Campagnolo, Angiogenesis in Tissue Engineering: As Nature Intended? Frontiers in Bioengineering and Biotechnology, 2020. 8(188). 